\title{
IPSID- a Neglected Cause of Chronic Diarrhea
}

\author{
Md. Rubel Miah', Syed Ali Imam², Sultana Akter ${ }^{3}$, Ejrarul Alam Khan ${ }^{4}$, Md. Uzzwal Mallik ${ }^{5}$, A.K.M. Humayon \\ Kabir ${ }^{6}$, Sarmistha Biswas ${ }^{6}$, Md. Asadul Kabir7, Md. Hafiz Sardar ${ }^{7}$
}

\begin{abstract}
:
immunoproliferative small intestinal disease is considered as a rare condition and has endemicity in Mediterranean countries and often associated with campylobacter jejuni infection. This condition varies in severity from benign to frankly malignant. Prolonged remission can be obtained with long term antibiotic therapy but chemotherapy is required for those who have aggressive disease. Bangladeshi data on IPSID is sparse. Here our patient 21 years old male presented with chronic diarrhoea, occasional vomiting, severe weakness and significant unintentional weight loss. He had cachectic body built, bipedal edema, easy bruising, glossitis with angular stomatitis. His upper Gl endoscopy showed multiple nodular lesion in the duodenum. Biopsy was taken from nodular lesion and sent for histopathology, geneXpert and culture. Histopathology revealed IPSID. He was treated with tetracycline and improved clinically.
\end{abstract}

Key words: ipsid, chronic diarrhoea,duodenal nodules

\begin{abstract}
(c) (1)(2) (2)
DOI: https://doi.org//0.3329/jom.v23il.57942 Copyright: (C) 2022 .Miah MR. This is an open access article published under the Creative Commons Attribution-NonCommercial-NoDerivatives 4.0 International License, which permits use, distribution and reproduction in any medium, provided the original work is properly cited, is not changed in any way and it is not used for commercial purposes.
\end{abstract}

Received: I5 November, 202I;

Accepted: 25 December, 2021

\section{Introduction :}

Unique form of small bowel lymphoma diffusely involving the entire intestine was first described in Oriental Jews and Arabs and is referred to as immunoproliferative small intestinal disease (IPSID), Mediterranean lymphoma or alpha heavy chain disease. This is a B cell tumor.The disease usually occurs in the second or third decade of life although has been observed in older individuals. Curious features in many patients with IPSID is the presence in the blood

and intestine of secretion of an abnormal immunoglobulin A that contains a shortened Alpha Heavy Chain and is devoid

1. Junior consultant, Dept of Medicine, Dhaka Medical College Hospital

2. Asst. Registrar, Dept of Medicine, Dhaka Medical College Hospital

3. Intern doctor, Dept of Medicine, Dhaka Medical College Hospital

4. Registrar, Dept of Medicine, Dhaka Medical College Hospital

5. Asst. Professor, Dept of Medicine, Dhaka Medical College Hospital

6. Associate Professor, Dept of Medicine, Dhaka Medical College Hospital

7. Professor, Dept of Medicine, Dhaka Medical College Hospital Corresponding author: Dr. Md Rubel Miah, Junior Consultant, Dept of Medicine, Dhaka Medical College Hospital, Dhaka. Email: rubelmd22@gmail.com of light chains are produced by plasma cells infiltrating the small bowel.

The clinical course of patient with IPSID is generally one of exacerbation and remission with death frequently resulting from either progressive malnutrition and wasting or the development of an aggressive lymphoma. The use of oral antibiotics such as tetracycline appears to be beneficial in early phases of disorder suggesting a possible infectious etiology.

Combination chemotherapy has been administered at advance stages of the disease with variable results. As IPSID is a malignant disease and can be wholly treated with simple antibiotic if diagnosed earlier,so we should consider it as an important differential during evaluation of a patient with chronic diarrhea.

\section{Case summary:}

A 21 years old male, cobbler, married, normotensive and non diabetic, smoker, nonalcoholic hailing from Jurain, Dhaka presented with frequent passage of loose stool initially 2-3 times per day but gradually increased to around 6-8 times per day with nocturnal episode for one and a half years. Stool was semi solid to watery, voluminous, not frothy, greasy, foul smelling, not mixed with blood or mucus and no relation with dairy, wheat products or drugs. The patient 
also experiences nausea with occasional vomiting and abdominal fullness. He also complain of severe weakness and lethargy with significant unintentional weight loss about $15 \mathrm{~kg}$ over the course of illness. There is no history of fever, abdominal pain, joint pain, skin rash, oral ulceration, palpitation, sweating, tremor, tingling or numbness, abdominal surgery, radiation exposure. He has no history of contact with TB patient and no history of blood transfusion or extra marital sexual exposure. There is no history of such illness in his family. He belongs to a low socio economic background and gives history of poor sanitation. His physical examination revealed cachectic body built, mild anaemia, bipedal edema, easy bruising, glossitis with angular stomatitis.On investigation, his complete blood count revealed normal total and differential count. Hemoglobin level was $15.7 \mathrm{gm} / \mathrm{dL}$, serum creatinine $0.89 \mathrm{mg} / \mathrm{L}$, magnesium $2.10 \mathrm{mg} / \mathrm{L}$, stool RME and CS revealed color-Brown, consistency - loose, RBC- nil, mucus- nil, pus cell 3-6/HPF, sensitivity- no growth, fecal fat estimation -negative, fecal calprotectin - $10 \mathrm{mcg} / \mathrm{gm}$, Mountax test - $4 \mathrm{~mm}$, chest Xray PA view- normal, USG whole abdomen -normal study, tTG(tissue transglutaminase) - $10.2 \mathrm{unit} / \mathrm{ml}$, anti-HIV 1 and 2- negative, vitamin D $5.2 \mathrm{ng} / \mathrm{L}, \mathrm{PT}$ - 13 seconds and serum amylase - 46 unit/L, serum lipase - 44 unit/L, serum vitamin B12 - $310 \mathrm{ng} / \mathrm{L}$, serum folate - $2.2 \mathrm{ng} / \mathrm{mL}$. Colonoscopy revealed normal study, upper GI endoscopy showed multiple nodular lesion in the distal duodenum(Figure 1) and biopsy from distal duodenum revealed the villi are short and broad, lamina propria contains many large Lymphocytes, plasma cells, small number of eosinophil and a few lymphoid follicles. The surface epithelium shows invasion by Lymphocytes, suggestive of immunoproliferative small intestinal disease (IPSID) (Figure 2).

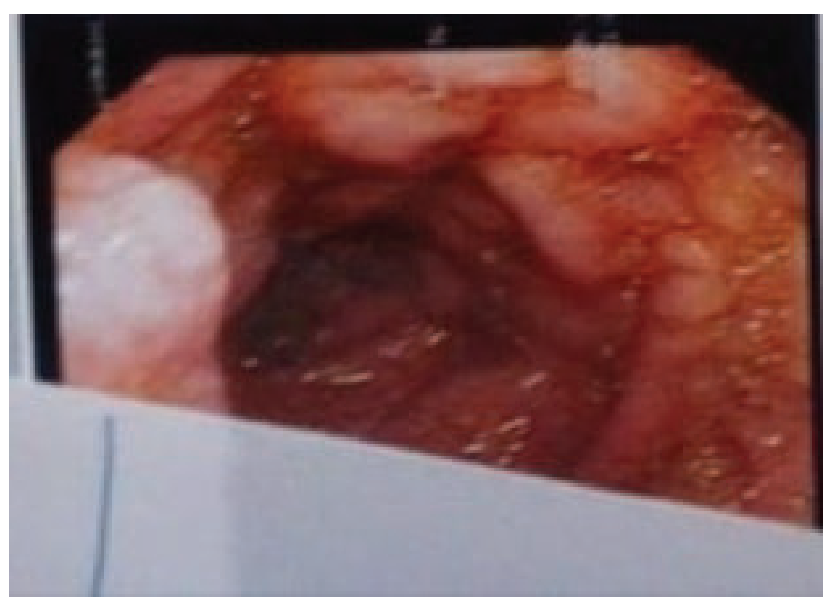

Fig.-1: Endoscopic picture showing multiple nodules in the duodenum

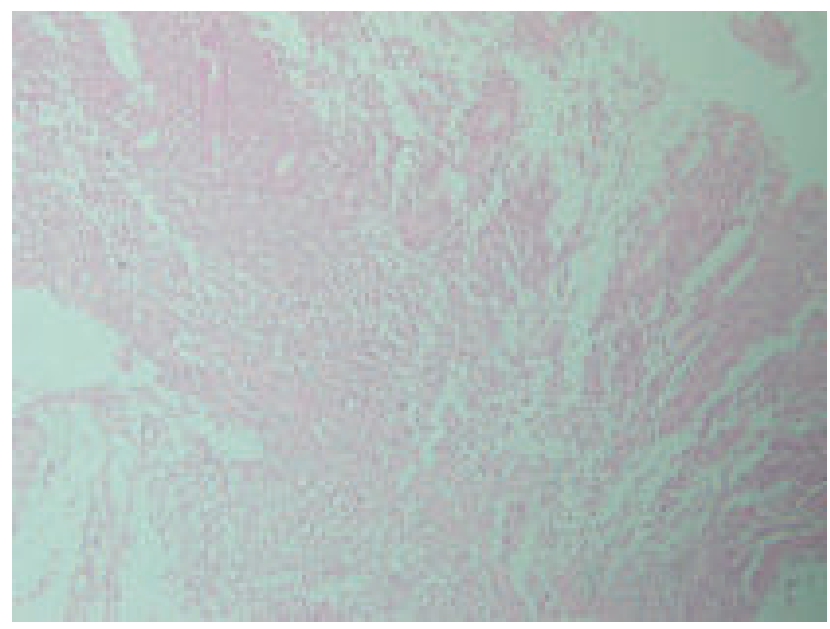

Fig.-2: Microscopic view of lamina propria containing multiple large lymphocytes, plasma cells and few eosinophils

Serum protein electrophoresis revealed alpha heavy chain, CT scan of whole abdomen showed multiple enlarge centrimetric and subcentimetric lymph nodes noted in paracaval and mesenteric region, dilated and matted small bowel loops, barium follow through gives normal impression. Patient was treated with tetracycline 12 hourly for 6 months and showed significant improvement with reduction of bowel frequency and weight gain.

\section{Discussion}

IPSID is confined to certain regions of the world, especially North Africa, Israel, and surrounding Middle Eastern and Mediterranean countries. ${ }^{1}$ IPSID is seen less often in other areas, including Central and South Africa, India and East Asia, and South and Central America. Patients usually present with chronic diarrhoea, colicky abdominal pain, anorexia and significant weight loss. The duration of symptoms is often months to years. The diarrhoea initially may be intermittent but becomes voluminous and foul smelling and malabsorption develops. About half of the patient had fever. Physical examination reveals evidence of malnutrition, digital clubbing and peripheral edema. Late physical manifestations are ascites, hepatosplenomegaly, abdominal mass and peripheral lymphadenopathy.

Endoscopy may reveal thickened mucosa, nodules or evidence of submucosal infiltration, rendering the intestine immobile, tender and non distensible. Patients are frequently anemic because of vitamin B deficiency and erythrocyte sedimentation rate is elevated in one third of cases. The circulating Lymphocytes count is low, campylobacter jejuni has been implicated in a high percentage of patients by PCR DNA Sequencing. Serum IgG and IgM levels may be high or low; Ig A levels are usually low or undetectable. Gross lesions 
are generally confined to proximal small intestine with adenopathy of adjacent mesenteric nodes. Although some patients have thickening of mucosal fold only, others have a generalized thickening of bowe wall, discrete masses, nodules or polypoid lesions. Although grossly only the proximal bowel wall is involved, histopathologically the disease is characterized by a dense mucosal and submucosal cellular infiltrates that extends continuously throughout the length of the small intestine. Various pathologic staging systems have been proposed. 8,9 The early infiltrate broadens villi and shortens and separates crypts but epithelial cells remain intact. Mesenteric lymph nodes are enlarged in early lesions. Patient with early disease (Salem stage 0 disease) is generally treated with antibiotics for six months or more. The two most commonly used regimens are tetracycline alone and a combination of ampicillin and metronidazole . Response rates have ranged from 33 to $71 \%{ }^{13}$; in one study the complete response rate was $71 \%$ with disease-free survival of $43 \%$ at 5 years $^{11}$. Observation suggesting that IPSID may be initiated by an infectious agent includes ${ }^{2}$ : an association of the disease with lower socioeconomic status and poor sanitation; a high prevalence of intestinal bacterial overgrowth and parasitosis; a decrease in disease incidence when living conditions have improved in endemic areas, and a response of early lesions to antibiotic therapy. High index of suspicion is required to diagnose IPSID and more malignant appearing histology may be present only in deeper layer so laparatomy with full thickness intestinal biopsy and biopsy of mesenteric lymph nodes should be considered despite normal endoscopic biopsies if suspicion for IPSID is $\operatorname{high}^{10}$.

\section{Conclusion}

IPSID is a primary small intestinal lymphoma, possibly caused by infective etiology and can be treated with simple antibiotic with an expected outcome if diagnosed early. So as its management gets easier if diagnosed earlier and it is not uncommon in our settings, we should consider it as important differential when we are dealing with particularly a young patient with chronic diarrhoea and malabsorption.

\section{Conflict of interest: None}

\section{References}

1. Salem PA, Estephan FF. Immunoproliferative small intestinal disease: Current concepts. Cancer J 2005; 11:374-82.

2. Suarez F, Lortholary O, Hermine O, et al. Infection associated lymphomas derived from marginal zone B cells: A model of antigen-driven lymphoproliferation. Blood 2006; 107: 3034-44.

3. Al-Saleem T, Al-Mondhiry H. Immunoproliferative small intestinal disease (IPSID): A model for mature B-cell neoplasms. Blood 2005; 105:2274-80.

4. Lecuit M, Abachin E, Martin A, et al. Immunoproliferative small intestinal disease associated with Campylobacter jejuni. N Engl J Med 2004; 350:239-48.

5. Seligmann M, Danon F, Hurez D, et al. Alpha-chain disease: A new immunoglobulin abnormality. Science 1968; 162: 1396-7.

6. saacson PG, Price SK. Light chains in Mediterranean lymphoma. J Clin Pathol 1985; 38:601-7.

7. Smith WJ, Price SK, Isaacson PG. Immunoglobulin gene rearrangement in immunoproliferative small intestinal disease (IPSID). J Clin Pathol 1987; 40:1291-7.

8. Salem P, el-Hashimi L, Anaissie E, et al. Primary small intestinal lymphoma in adults. A comparative study of IPSID versus non-IPSID in the Middle East. Cancer 1987; 59: 1670-6.

9. Galian A, Lecestre MJ, Scotto J, et al. Pathological study of alpha chain disease, with special emphasis on evolution. Cancer 1977; 39:2081-101.

10. Martin IG, Aldoori MI. Immunoproliferative small intestinal disease: Mediterranean lymphoma and alpha heavy chain disease. Br J Surg 1994; 81:20-4.

11. Akbulut H, Soykan I, Yakaryilmaz F, et al. Five-year results of the treatment of 23 patients with immunoproliferative small intestinal disease: A Turkish experience. Cancer 1997; 80:8-14.

12. Ben-Ayed F, Halphen M, Najjar T, et al. Treatment of alpha chain disease. Results of a prospective study in 21 Tunisian patients by the Tunisian-French intestinal Lymphoma Study Group. Cancer 1989; 63:1251-6.

13. Fine KD, Stone MJ. Alpha-heavy chain disease, Mediterranean lymphoma, and immunoproliferative small intestinal disease: A review of clinicopathological features, pathogenesis, and differential diagnosis. Am J Gastroenterol 1999; 94:1139-52.

14. el Saghir NS. Combination chemotherapy with tetracycline and aggressive supportive care for immunoproliferative smallintestinal disease lymphoma. J Clin Oncol 1995; 13:794-5.

15. Salimi M, Spinelli JJ. Chemotherapy of Mediterranean abdominal lymphoma. Retrospective comparison of chemotherapy protocols in Iranian patients. Am J Clin Oncol 1996; 19:18-22. 